\title{
A epidemiologia na avaliação da qualidade: uma proposta 1
}

\author{
Epidemiological standards for assessing quality: \\ a proposal 1
}

\footnotetext{
1 Trabalho baseado em dissertação de mestrado apresentada à Faculdade de Saúde Pública,

Universidade de São Paulo.

2 Núcleo de Investigação em

Saúde da Mulher e da

Criança, Instituto de Saúde

Rua Santo Antonio 590

2 o andar, São Paulo, SP

01314-000, Brasil.

Fax: (011) 6052772
}

Abstract This paper proposes a strategy for defining indicators to assess the quality of the process of health care, based on the identification of the objectives of the care being delivered and on the verification of whether the technical guidel ines for the procedures are being enforced. The article goes on to apply the proposed methodol ogy to the process of health care during childbirth, based on a perspective of comprehensive care and adopting both an individual and collective risk approach. It uses a data bank of 4,558 Simplified Perinatal Clinical Histories (CLAP/PAHO/WHO) from 12 hospitals. The indicators sel ected were: V.D.R.L., Rh blood typing, antitetanus vaccination, rate of cesarean sections, reception of the baby by a pediatrician at birth, Apgar score, evaluation of the gestational age by physical exam, rooming-in, exclusi ve breastfeeding upon discharge, and referral for a puerperal consultation. Methods for classification of hospitals were the sum of their scores for each indicator, gold standard, and ranking. This methodology warranted identification of one hospital with excellent quality of care, five with good care, two with fair care, and four whose performances were completely unsati ffactory. Finally, some remarks on the assessment of quality of health care are made and future devel opments are proposed.

Key words Epidemiology; Health Services; Perinatal Care; Quality of Health Care; Evaluation Studies

Resumo Este artigo propõe uma estratégia de defini ção de indicadores para aval iar a qual idade do processo de assi stência, baseada na identificação dos objeti vos dessa assistência e na verifi cação da existência de normas técni cas que a nortei em. Simultaneamente, aplica esta metodologia ao processo de assistência ao nascimento e parto, adotando enfoque de risco, tanto para a saúde individual, como para a coletiva. Utiliza para tal um banco de dados de 4.558 Histórias Clínicas Perinatais Si mpli fi cadas de 12 hospitais. Os indi cadores selecionados foram: sorologia para sífilis, tipagem sanguínea Rh, vacinação antitetânica, taxa de cesáreas, recepção do bebê por pediatra na sala de partos, boletim de Apgar, avaliação de idade gestacional por exame físi co, alojamento conj unto, alei tamento materno exclusi vo na alta, encaminhamento para consulta de puerpério. Para classificação dos hospitais, foram utilizadas a soma si mples, padrão-ouro, e hi erarquização. A metodologia proposta permitiu identificar um hospital com qualidade excelente, cinco com qualidade boa, dois com qualidade regular e quatro com desempenho completamente insatisfatório.

Palavras-chave Epidemiologia; Serviços de Saúde; Avaliação da Qualidade dos Cuidados de Saúde; Assi stência Perinatal; Obstetrícia 
Introdução

Uma primeira dificuldade que se apresenta quando se deseja avaliar a qualidade é sua definição conceitual. De acordo com Vuori (1991), "o termo qualidade geralmente denota um grande espectro de características desejáveis de cuidados que incluem a efetividade, eficácia, eficiência, eqüidade, aceitabilidade, acessibilidade, adequação e qualidade técnicocientífica". Portanto, avaliar a qualidade da assistência é um procedimento complexo, que demanda, por vezes, conhecimentos ainda não disponíveis e que precisam ser desenvolvidos.

Para estimar a qualidade dos serviços, Donabedian (1988) identificou três abordagens: estrutura, processo e resultado. As medidas de estrutura envolvem informações sobre recursos físicos, humanos, materiais, formas de organização e funcionamento (normas e procedimentos), tipo e especialização de equipamento etc. Para a avaliação da estrutura dos serviços de assistência ao nascimento e parto, há um documento da OMS/OPAS (1987) de avaliação dos serviços de assistência à população materno-infantil, que abrange desde ambulatório em unidades básicas até hospitais de referência; há também um documento anterior, da Secretaria de Estado da Saúde de São Paulo (s/ data), com as normas de acreditação dos serviços de berçário. O resultado se refere ao efeito que ações e procedimentos tiveram sobre o estado de saúde dos pacientes, ou, no caso, o binômio mãe-filho. Tradicionalmente, segundo Elinson (apud Lewis, 1974), óbitos, patologias, incapacidades, desconforto e insatisfação têm sido adotados como indicadores de resultados. Na avaliação da assistência perinatal, o indicador mais utilizado tem sido o coeficiente de mortalidade perinatal (BobadilIa, 1989; Holthof \& Prins, 1993; Thompson et al., 1968; Tokuhata et al., 1973; Williams, 1979). Já a avaliação da qualidade do processo, ou se$\mathrm{ja}$, as atividades realizadas pelos provedores de assistência, tanto diagnósticas, como terapêuticas e de reabilitação, apresenta algumas complexidades, uma vez que pode referir-se tanto ao componente técnico, como ao de relação interpessoal, este com dificuldades ainda maiores de mensuração. No caso dos serviços perinatais, o que poderia ser medido? Bobadilla (1989), apesar de propor vários indicadores de qualidade, para processo usou apenas a taxa de cesáreas do hospital. Outros autores nem chegaram a explicitar quais indicadores representariam o processo (Holthof \& Prins, 1993; Thompson et al., 1968; Tokuhata et al., 1973; Williams, 1979), trabalhando principalmente com dados de estrutura e características da demanda. Uma importante constatação da literatura sobre processo é que a maioria dos autores adota uma perspectiva reducionista do processo de assistência, primeiro individualizando cada procedimento, e em seguida decompondo-o nas suas várias partes.

Este trabalho se propõe a sugerir indicadores para esta avaliação adotando um outro paradigma de processo de assistência, que incorpora uma perspectiva holística e a visão de processo como uma continuidade. Parte, ainda, de uma necessidade de também incorporar a dimensão coletiva, adicionando uma abordagem de risco coletivo à assistência individual. O enfoque de risco tem sido muito utilizado nessa dimensão individual, principalmente para a descrição da etiologia (Plaut, 1984). É necessário resgatá-lo para a dimensão do coletivo. Carval heiro (1984) sustenta que "avaliar o processo-saúde-doença em sua dimensão coletiva não pode ser feito somente com a dimensão da epidemiologia tradicional (tempo-lugar-pessoa) se não se busca a essência do processo". Embora não se trate aqui de uma instância do processo saúde-doença, foi-se buscar a essência da assistência ao nascimento e parto nos objetivos do Programa de Assistência MaternoInfantil e nas múltiplas normas que o complementam na orientação do que é uma assistência desejável. Afinal, as normas técnicas representam um conhecimento científico acumulado, um saber reconhecido e aceito nos meios científicos, ainda que nem sempre sejam atualizadas na velocidade em que progride esse conhecimento. Também identificou-se uma necessidade de indicadores que fossem aplicáveis a todas as maternidades, independentemente de seu porte, movimento, ou complexidade. Tendo em mente todas essas considerações, este trabalho foi realizado com os objetivos de a) testar indicadores derivados de normas e vinculados aos objetivos do programa de assistência materno-infantil como potenciais instrumentos de avaliação da qualidade do processo de assistência ao nascimento e parto, para b) verificar o quanto esta metodologia se adequa à elaboração de indicadores de qualidade de processo.

\section{Material e métodos}

\section{Material}

Os dados são de um estudo transversal de 12 hospitais da Região Metropolitana de São Paulo, que integraram a pesquisa “História Clínica 
Perinatal Simplificada: É viável em São Paulo?", realizada por Rattner \& Segre (1990). Esta objetivou identificar os fatores de risco de morbidade e mortalidade perinatais na população desses hospitais e testar a viabilidade de uso do formulário "História Clínica Perinatal Simplificada" (HCPS), proposto por Belizan et al. (1976) e depois explicitado por Diaz et al. (1990). Também propunha testar a viabilidade de sua utilização a partir de seu preenchimento por profissionais não-médicos. Os dados referem-se a 4.558 nascimentos e altas de recémnascidos ocorridos entre 10 de dezembro de 1988 e 15 de fevereiro de 1989. A estratégia de captação foi um convite para integrar o projeto a 12 hospitais públicos ou filantrópicos. Dois declinaram, por estarem utilizando outro sistema de informação; em conseqüência, foram convidados dois hospitais de convênio, que acederam. Indicadas pela direção, duas profissionais de cada hospital foram submetidas a um treinamento de 16 horas sobre a coleta de dados, com uma avaliação final. Também foi elaborado e distribuído um manual de preenchimento da HCPS. Os formulários preenchidos foram supervisionados por quatro profissionais de nível universitário. Em reuniões quinzenais, a coordenação esclarecia as dúvidas das supervisoras e orientava quanto a problemas de preenchimento. Quando estes eram muito freqüentes, a coordenação visitava o hospital para consulta direta aos prontuários médicos. A digitação utilizou o programa SIPSistema Informático Perinatal de Simini et al. (1989), versões 2.4 e 5.5, elaborado pelo Centro Latino-Americano de Perinatologia-CLAP.

\section{Seleção dos indicadores}

De acordo com Reis (1971), os objetivos do Programa de Atenção Materno-Infantil são: a) atenção pré-natal precoce e adequada assistência ao parto e puerpério, em condições de evitar os riscos e o choque emocional; b) exame pós-parto; c) exame e assistência ao recémnascido; d) estímulo ao aleitamento natural e à mais íntima convivência do lactente com sua mãe. Incluem-se todas as atividades de promoção e recuperação da saúde materno-infantil. Para atingir esses objetivos, o Ministério da Saúde (1984a, 1984b, 1991a, 1991b) elaborou o Programa de Assistência Integral à Saúde da Mulher (PAISM) e o Programa de Assistência Integral à Saúde da Criança (PAISC), que tiveram a sua contrapartida pelo Governo do Estado de São Paulo (SES/SP, 1975, 1985).

Segundo Cooke (1988), a alta probabilidade de morte que existe ao redor do momento do nascimento não se assemelha à de nenhuma outra etapa da vida. Deve-se à vulnerabilidade do feto, ao stress físico e ao trauma derivado do nascimento. O hospital que presta assistência ao nascimento e parto pode ser considerado o "locus" privilegiado para a avaliação da assistência, já que é onde se realiza o clímax de nove meses de expectativa. Seu desempenho será determinado pelo conhecimento dos fatos, problemas e da assistência que precedeu seu atendimento. Também será fundamental na determinação das condições de vida e saúde do binômio mãe-filho após a alta. Daí resulta a necessidade de uma visão de integralidade do processo, que tome em consideração aspectos prévios à admissão da gestante (o período prénatal), aspectos da própria prestação de serviços intra-hospitalares e aspectos referentes ao manejo do binômio mãe-filho após a alta. Convém que o hospital que presta assistência ao nascimento e parto manifeste sua preocupação com todos esses aspectos e é por meio da proporção com que essa preocupação aparece em seus registros que se poderá inferir a qualidade de seus serviços.

Em decorrência dessa perspectiva e considerando os objetivos já citados para o Programa de Atenção Materno-Infantil (Reis, 1971), foram identificados os seguintes potenciais indicadores da qualidade da assistência hospitalar ao nascimento e parto, cujos dados encontravam-se disponíveis no formulário da HCPS: Atenção pré natal precoce: 1) sorologia para sífilis; 2) tipagem sanguínea Rh; 3) vacinação anti-tetânica.

Adequada assistência ao parto: 4) taxa de cesáreas do hospital.

Exame eassistência ao recém-nascido: 5) recepção do recém-nascido por médico, na sala de partos; 6) boletim de Apgar no primeiro minuto; 7) avaliação da idade gestacional por exame físico.

Estímulo ao aleitamento natural eà mais íntima convivência do lactente com sua mãe: 8) existência de alojamento conjunto; 9) proporção de altas com aleitamento materno exclusivo.

Exame pós-parto: 10) proporção de mães com encaminhamento para consulta de puerpério.

Considerações sobre cada um desses indicadores são tecidas de forma mais elaborada em Rattner (1991). Cabe ressaltar, entretanto, que estes foram selecionados como indicadores de qualidade, pois:

- implicam procedimentos propedêuticos, de diagnóstico ou de encaminhamento, cuja inobservância ou uso inadequado acarretam riscos, tanto no nível individual, para o binô- 
mio mãe-filho, quanto no nível do coletivo, afetando coeficientes de morbidade e mortalidade populacionais;

- são tecnologias acessíveis, que deveriam estar disponíveis em todos os hospitais;

- implicam procedimentos de baixo custo, com alta relação custo/ benefício;

- representam uma visão de integralidade do ciclo gravídico-puerperal;

- em sua maior parte, representam procedimentos normalizados, seja pelo Ministério da Saúde (1984a, 1984b, 1986, 1991a, 1991b), pelo Ministério da Previdência e Assistência Social (1983), seja pela Secretaria de Saúde do Estado de São Paulo (s/ data, 1975, 1984, 1985, 1989, 1994);

- são em número reduzido, assim simplificando os procedimentos de avaliação de qualidade do processo de assistência ao nascimento e parto.

\section{Metodologia de análise}

Tratamento dos dados e interpretação dos indicadores

Os indicadores 1, 2 e 3 (RSS, Rh eVAT) referemse à anamnese em relação à assistência pré-natal, e foram mensurados considerando-se a proporção dos prontuários em que constava essa informação, significando terem sido pesquisados e registrados. Nos indicadores 6 e 7 (Apgar e avaliação de idade gestacional), entendeu-se que, se o procedimento foi executado, ocorreu o respectivo registro. Os indicadores 4, 5, 9 e 10 demandaram o cálculo dessa atividade ou procedimento dentre as alternativas possíveis registradas. Para o Alojamento Conjunto, em vista do formulário utilizado não especificar se é integral ( 24 horas) ou sistema misto (alojamento conjunto durante o dia, berçário durante a noite), a verificação foi feita durante a visita. Se era integral, foram atribuídos cem pontos; se utilizava o sistema misto, cinqüenta pontos; e nenhum foi atribuído aos hospitais que apenas dispunham de berçário tradicional. Quanto ao indicador 4 - taxa de cesáreas -, para que se pudesse levar em conta sua variação segundo as características da demanda, foram consultados todos os formulários em que a cirurgia foi realizada e identificada a sua indicação. Quando não constava da HCPS, buscou-se a informação nos prontuários. Nos casos com duas ou mais indicações, foi selecionada a que mais fortemente determinou o procedimento. Saliente-se que a indicação "uma cesárea anterior" foi considerada como "sem indicação". Através destes procedimentos, a taxa de cesáreas foi transformada em "proporção de cesáreas explicadas pelos registros". Esta estratégia serviu não apenas para converter este indicador em um que variasse entre 0 e 100, como também para incorporar outras informações que reduzissem a possibilidade de julgamento subjetivo (taxa alta, média, inadequada etc). A Tabela 1 sumariza a estratégia de construção desse indicador. Finalmente, foi realizada a adequação de denominadores dos indicadores: para a avaliação da idade gestacional, deixaram de ser computados os natimortos, e para as altas com aleitamento materno exclusivo, além dos natimortos, também os óbitos perinatais e materno.

\section{Métodos de avaliação}

Os dados foram trabalhados em vários estágios, na tentativa de validar-se esta forma de avaliação:

a) Soma: Inicialmente, para cada hospital foram somadas as proporções obtidas em todos os indicadores. Esta soma resultou numa classificação inicial, que orientou a ordem de apresentação dos hospitais para todos as outras avaliações.

b) Classificação dos hospitais de acordo com número de indicadores com valor superior a 80\%: Vuori (1980) afirma que muitas vezes o bom não se adequa ao ótimo e sugere que, no nível de um atendimento individual, este seja considerado "bom" se $80 \%$ dos critérios expli-

Tabela 1

Sumário da estratégia de construção do indicador Taxa de Cesáreas Explicada, 12 hospitais da Grande São Paulo, 01/12/1988 a 15/02/1989.

\begin{tabular}{|c|c|c|c|c|c|c|c|c|c|c|c|c|c|}
\hline & $A$ & $B$ & $\mathrm{C}$ & $\mathrm{D}$ & $E$ & $\mathrm{~F}$ & G & $\mathrm{H}$ & 1 & $J$ & K & $\mathrm{L}$ & Total \\
\hline Taxa de cesáreas & 32,1 & 44,6 & 31,5 & 35,1 & 41,6 & 18,7 & 11,8 & 47,3 & 21,1 & 54,0 & 55,1 & 28,1 & 31,6 \\
\hline Total de indicações & 82 & 125 & 119 & 115 & 84 & 71 & 43 & 75 & 59 & 34 & 89 & 255 & 1151 \\
\hline Total de cesáreas & 85 & 150 & 165 & 117 & 94 & 79 & 50 & 113 & 83 & 82 & 141 & 281 & 1440 \\
\hline Taxa explicada & 96,5 & 83,3 & 72,1 & 98,3 & 89,4 & 89,9 & 86,0 & 66,4 & 71,1 & 41,5 & 63,1 & 90,7 & 79,9 \\
\hline
\end{tabular}


citados forem satisfeitos. Numa adaptação desta afirmativa, neste trabalho foram computados em números absolutos os indicadores que apresentaram valores superiores a essa porcentagem.

c) Validação através de um padrão: Lembcke (1967), ao discorrer sobre auditorias, propôs, para o estabelecimento de padrões, o grau e percentagem de cumprimento dos critérios estabelecidos em hospitais docentes. Refere que assim "se constitui um padrão genuíno, que representa o que pode fazer-se bem, a qualquer momento, sem um incentivo especial". Propõe o cálculo do cumprimento dos critérios para cada hospital, usando-se o hospital docente como referência. Refere que, já que raramente se alcança a perfei ção, devem ser feitas concessões, sistemática e objetivamente, para adequar a falta de exati dão nos critérios e no exercício do julgamento, e sugere a adequação do padrão de $100 \%$, por exemplo, para $80 \%$, no que é referendado por Vuori (WHO, 1982). $\mathrm{Na}$ medida em que integra ao conjunto dos hospitais estudados um hospital universitário, este passou a ser o padrão deste processo de validação. Na prática, isto significou que todos os valores alcançados por esse hospital em todos os indicadores foram considerados equivalentes a $100 \%$, e os dos outros hospitais foram recalculados utilizando-se este hospital A como referência.

d) Validação através de hierarquização ("ranking"): Neste processo, tenta-se levar em consideração as condições ou circunstâncias reais de execução das atividades. Assim, para cada indicador foi identificado o hospital que apresentava o valor mais el evado, ao qual foi atribuído o valor 100. Ao de valor menos adequado, foi atribuído zero ponto, e os hospitais foram-redistribuídos nessa nova escala, a exemplo do procedimento utilizado por Guedes (1972).

e) Classificação: Em cada um dos processos de avaliação adotados, os hospitais foram classificados em ordem decrescente. A Tabela 3 sumariza os resultados dos vários métodos, assim como propõe uma classificação e pontos de corte.

\section{Resultados}

Os indicadores propostos permitiram detectar vários problemas. Por exemplo, patologias que se constituem em risco tanto coletivo quanto individual, como o tétano e a sífilis, de uma maneira geral não são preocupação presente entre os que prestam assistência médica individual.
A sífilis foi pesquisada em menos da metade das pacientes ( $45,7 \%)$, com a alta prevalência de $3,3 \%$ de resultados positivos (Rattner \& Segre, 1990), sendo que apenas quatro hospitais pesquisaram mais de $80 \%$ de suas pacientes. Sífilis é uma doença de transmissão sexual, cuja cadeia poderia ser interrompida caso se aproveitasse a ocasião do contato da parturiente com o sistema de saúde para a realização de seu diagnóstico e tratamento (praticamente todos os partos são institucionais no Estado), com vantagens óbvias para o casal e seu bebê (Garland \& Kelly, 1989).

Em apenas 5\% dos prontuários houve registro de vacinação antitetânica. No momento da internação, já não teria ocorrido a prevenção do tétano materno e/ ou neonatal; entretanto, poder-se-ia usar a oportunidade para iniciar o esquema vacinal ou aplicar um reforço na mãe.

Williams et al. (1988) alertam para o risco de oportunidades perdidas na ocasião do contato de clientes com os serviços de saúde, e estes dois indicadores ilustram bem como estas podem se dar. Mesmo nas internações por aborto ou natimorto, as pacientes deveriam ser investigadas e ter sua profilaxia encaminhada, já que a sífilis pode determinar ambas as perdas. Vale lembrar que estas duas patologias estão relacionadas na versão preliminar de um documento da OPAS sobre qualidade em serviços de saúde reprodutiva (Mora et al., 1993). A preocupação com o risco de isoimunização Rh, entretanto, parece estar presente, pois cerca de $80 \%$ de todas as pacientes foram pesquisadas, e proporções superiores a $80 \%$ de pacientes foram encontradas em dez dos 12 hospitais.

As taxas de cesárea estiveram entre $11,8 \%$ e $55,1 \%$ (hospitais G e K), variação de quase cinco vezes, e a proporção de cirurgias explicadas foi $80 \%$, com variação entre $41,5 \%$ e $98,3 \%$, evidenciando que boa parte das cirurgias poderia ter sido evitada. Ressalte-se que este não é um procedimento isento de riscos (Nielsen \& Hokegard, 1984), que há hospitais no Estado de São Paulo com taxas ainda mais altas, entre $80 \%$ e $100 \%$, e que a taxa do Estado tem se mantido por volta de 48\% desde 1987 (Rattner, 1996), isto é, bem superior aos $31,6 \%$ de cesáreas nesta população, achado que certamente se deve à proporção de hospitais públicos neste estudo. Gentile de Mello (1977), um pioneiro no estudo da epidemiologia das cesáreas em nosso País, apontava para a grande disparidade que encontrou em 1971: taxas entre 7\% e $13 \%$ nos hospitais públicos e valores superiores a $30 \%$ na rede contratada, o que atribuía ao regime de livre-escolha e pagamento por unidade de serviço. No levantamento realizado 
Tabela 2

Resultados dos indicadores nos hospitais (\%) e respectiva classificação, 12 hospitais da Grande São Paulo, 01/12/88 a 15/02/89.

\begin{tabular}{|c|c|c|c|c|c|c|c|c|c|c|c|c|c|}
\hline Variáveis & A & B & C & $\mathrm{D}$ & $E$ & $F$ & G & $\mathrm{H}$ & 1 & $J$ & $K$ & $L$ & Total \\
\hline \multirow[t]{2}{*}{ RSS } & 87,2 & 64,0 & 90,8 & 42,4 & 80,6 & 33,3 & 98,8 & 2,5 & 50,5 & 11,2 & 17,6 & 2,5 & 45,7 \\
\hline & 30 & 5응 & 20 & 70 & 40 & 8응 & 10 & 11 & 6응 & 10 응 & 9응 & 110 & \\
\hline \multirow[t]{2}{*}{$\mathrm{Rh}$} & 99,2 & 96,4 & 94,6 & 90,7 & 97,4 & 91,3 & 99,0 & 91,6 & 78,3 & 80,3 & 89,8 & 31,6 & 78,8 \\
\hline & 10 & 40 & 50 & 80 & 3응 & 70 & 20 & 60 & $11^{\circ}$ & $10^{\circ}$ & 9응 & $12^{\circ}$ & \\
\hline \multirow[t]{2}{*}{ Vac. antitetânica } & 47,5 & 2,1 & - & 6,9 & 1,3 & 2,8 & 0,5 & - & 1,3 & 25,0 & 1,6 & 1,4 & 5,1 \\
\hline & 10 & 50 & $11^{\circ}$ & 30 & 80 & 40 & 10 & 110 & 80 & 20 & 60 & 70 & \\
\hline Tx de ces. & 96,5 & 83,3 & 72,1 & 98,3 & 89,4 & 89,9 & 86,0 & 66,4 & 71,1 & 41,5 & 63,1 & 90,7 & 79,9 \\
\hline explicada & 20 & 70 & 80 & 10 & 50 & 40 & 60 & $10^{\circ}$ & 9응 & $12^{\circ}$ & 110 & 30 & \\
\hline Médico & 98,1 & 97,9 & 78,6 & 94,9 & 92,5 & 95,8 & 1,5 & 97,9 & 1,0 & 19,7 & 1,2 & 0,3 & 48,6 \\
\hline na sala1 & 10 & 20 & 70 & 50 & 60 & 40 & 90 & $2^{\circ}$ & $11^{\circ}$ & 80 & $10^{\circ}$ & $12^{\circ}$ & \\
\hline \multirow[t]{2}{*}{ Apgar } & 100,0 & 99,7 & 99,6 & 99,1 & 96,5 & 93,9 & 94,6 & 99,6 & 88,9 & 77,0 & 81,2 & 97,8 & 95,1 \\
\hline & 1음 & 20 & 3응 & 50 & 7응 & 9응 & 8응 & 3응 & 10 응 & $12^{\circ}$ & 110 & 60 & \\
\hline Id. gest. por & 99,6 & 100,0 & 96,7 & 66,8 & 98,4 & 57,8 & 17,0 & 99,1 & 13,1 & 4,7 & 19,9 & 1,2 & 49,0 \\
\hline ex. físico 2 & 20 & 10 & 50 & 60 & 40 & 70 & 90 & 30 & $10^{\circ}$ & 110 & 80 & $12^{\circ}$ & \\
\hline Alojamento & 100,0 & 100,0 & 100,0 & 100,0 & 100,0 & 100,0 & 100,0 & 100,0 & 100,0 & - & - & 50,0 & 80,0 \\
\hline conjunto & 10 & 10 & 10 & 10 & 10 & 10 & 10 & 10 & 10 & 110 & $11 \stackrel{0}{2}$ & $10^{\circ}$ & \\
\hline Altas com & 92,6 & 64,2 & 83,6 & 87,8 & 33,3 & 94,0 & 66,9 & 97,0 & 94,9 & 95,2 & 0,4 & 89,7 & 78,8 \\
\hline aleit. mat. ${ }^{3}$ & 50 & 100 & 80 & 70 & 110 & 40 & 90 & 10 & 30 & 20 & $12^{\circ}$ & 60 & \\
\hline Enc. p/ cons. & 72,8 & 93,5 & 63,7 & 87,8 & 70,9 & 94,6 & 98,0 & - & 1,0 & 90,8 & 93,4 & - & 55,4 \\
\hline no puerpério & $7 \underline{0}$ & 30 & 9응 & 6음 & $8^{\circ}$ & 20 & 10 & 110 & $10^{\circ}$ & 50 & 4 & $11 \stackrel{0}{2}$ & \\
\hline \multirow[t]{2}{*}{ Total de pontos } & 893,5 & 801,1 & 779,7 & 774,7 & 760,0 & 753,4 & 662,3 & 654,1 & 500,1 & 445,4 & 368,2 & 365,2 & 616,4 \\
\hline & 10 & $2^{\circ}$ & 30 & 4 & 5은 & 60 & $7 \stackrel{0}{-}$ & $8^{\circ}$ & 9음 & $10^{\circ}$ & 11 & $12^{\circ}$ & \\
\hline $\begin{array}{l}\text { Total de } \\
\text { prontuários }\end{array}$ & 265 & 336 & 523 & 335 & 227 & 427 & 405 & 238 & 396 & 152 & 256 & 998 & 4558 \\
\hline
\end{tabular}

1 - 24,5\% dos prontuários sem nenhum registro.

2 - retirados do denominador os nascidos mortos.

3 - retirados do denominador natimortos, óbitos neonatais e materno.

nos prontuários, detectou-se uma grande proporção de indicações de sofrimento fetal, toxemias e distócias, fato certamente "epidêmico" em nossas maternidades, e que mais de $10 \%$ das mulheres submetidas a cesárea reportavam duas ou três cirurgias anteriores. Bobadilla (1989), ao avaliar a qualidade de hospitais mexicanos, utilizou taxa de cesáreas como seu único indicador de processo, sugerindo que este pode ser um excelente indicador de qualidade, ainda quando não se investigam as indicações da cirurgia.
A presença de pediatra no momento do nascimento ficou entre zero e praticamente $100 \%$, mostrando a grande variação existente. A maior parte dos $24,5 \%$ de ausência de qualquer registro no prontuário era proveniente de hospitais em que o recém-nato era recepcionado por auxiliar de enfermagem. Cabe sal ientar que este estudo é anterior às determinações oficiais que prevêem a remuneração do profissional (MS, 1993), e que é possível que hoje este quadro esteja alterado. Como não há medidas de resultado, seja de mortalidade perinatal ou neonatal 
precoce, não foi verificada a associação entre estas e a presença ou ausência do profissional no momento do nascimento. Mesmo assim, dado que há remuneração prevista pelo SUS, hoje em dia este indicador torna-se ainda mais importante, já que permite verificar quanto a determinação oficial está sendo cumprida.

O bol etim de Apgar encontrava-se registrado em 95\% dos prontuários, e apenas um hospital o registrava abaixo de $80 \%$ (hospital J), sugerindo que essa avaliação já foi incorporada à prática cotidiana da maior parte das maternidades, havendo reconhecimento de sua importância e de seu valor prognóstico. Cabe investigar se esse achado é generalizável, visto que este foi apenas um estudo piloto. Já a avaliação da idade gestacional por exame físico foi realizada em menos da metade de todos os bebês, variando entre $1,2 \%$ e $100 \%$ (hospitais $L$ e $B$ ), mostrando que este tipo de prática ainda não era corrente à época. É possível que, com o reconhecimento da importância do neonatologista e pagamento de seus serviços, este quadro tenha se alterado, e que este importante exame prognóstico tenha sido incorporado à rotina dos serviços.

Dos hospitais estudados, nove oferecem o Alojamento Conjunto integral e um adota o sistema misto, seguindo as recomendações de organismos internacionais (WHO, 1986) e órgãos oficiais (SES-SP, 1984; MPAS, 1983; MS, 1991b). Por outro lado, as altas em al eitamento materno exclusivo foram superiores a $80 \%$ em oito hospitais, porém apenas $78,8 \%$ na totalidade, sendo que em um deles (Hospital K) parece ser rotina a prescrição da mamadeira na alta. É sabido que o alojamento conjunto facilita o estabelecimento precoce do vínculo mãe-filho. Amamentar é um passo na prevenção da mortalidade pós-hospitalar, e profissionais de saúde têm um papel fundamental no incentivo a esta prática, embora nem todos tenham consciência disso (Hull et al., 1989). Na medida em que estas práticas ainda não estão generalizadas, os dados deste estudo suportam a adoção destes indicadores como refletindo a preocupação com o bem-estar do binômio.

Sabe-se que algumas das complicações pós-parto não são aparentes no puerpério imediato. A preocupação com a avaliação final da assistência prestada, para detecção de eventuais problemas e seu devido encaminhamento, deveria ser prática habitual dos serviços, assim como seu registro no prontuário. Entretanto, o encaminhamento para consulta de puerpério ocorreu em apenas $55,4 \%$ dos partos estudados, variando de zero (hospitais $\mathrm{H}$ e L) a 98\% (hospital G). Embora o ciclo gravídico- puerperal seja um "continuum" que se inicia com a concepção e termina ao final do puerpério, abrangendo os eventos intermediários de nascimento e parto, aparentemente algumas instituições o tratam como se fossem episódios envolvendo assistência não integrada: o prénatal, o parto com o nascimento e o puerpério. É raro todos esses períodos receberem assistência de uma mesma instituição, e os mecanismos de referência e contra-referência são negligenciados. Este indicador traduz tanto a preocupação dos profissionais com a continuidade da assistência após a internação, como a consciência da instituição de seu papel no sistema de saúde.

A Tabela 3 sumariza os resultados dos vários métodos utilizados, atribui uma avaliação aos hospitais, e propõe valores de corte arbitrários, mas que tomam em consideração a maneira com que os pontos dos hospitais se agruparam (tabelas para cada um dos procedimentos de avaliacão citados podem ser encontradas em Rattner, 1991). Utilizando como base a classificação obtida na soma simples de pontos, podese verificar que houve pequenas inversões ao adotar o padrão-ouro ou a hierarquização, mas que, em geral, houve consistência suficiente para que se pudessem definir grupos de hospitais. Através dos vários métodos, foi possível identificar um hospital de excelência, cinco hospitais com qualidade boa, dois com qualidade regular e quatro cujo processo, medido através dos indicadores propostos, mostrou-se insatisfatório.

\section{Discussão}

Este trabalho propõe critérios para a avaliação da qualidade do processo de assistência ao nascimento e parto. É uma proposta de utilização da Epidemiologia na avaliação da qualidade do processo dos serviços de assistência ao nascimento e parto, valorizando o enfoque de risco tanto individual, à mãe e seu concepto, como coletivo. Sífilis, isoimunização Rh e suscetibilidade ao tétano são riscos potenciais que a gestante pode trazer consigo ao ser internada. A cesárea, se procedimento desnecessário, ou inútil, como definido por Leape (1989), traz, por si só, riscos a ambos. A presença do pediatra na sala de partos, para a recepção do recém-nascido, visa a diminuir os riscos inerentes ao momento do nascimento. A avaliação imediata da vitalidade, através do Boletim de Apgar, e, no primeiro dia de vida, a da maturidade, ou seja, a avaliação da idade gestacional por exame físico, visam ao dimensionamento 
Tabela 3

Classificação dos hospitais segundo os diferentes métodos e atribuição de avaliação, 12 hospitais da Grande São Paulo, 01/12/88 a 15/02/89.

\begin{tabular}{|c|c|c|c|c|c|c|c|c|c|c|c|c|}
\hline & A & B & C & D & E & $\mathrm{F}$ & G & H & 1 & J & K & L \\
\hline \multirow[t]{2}{*}{ Soma dos pontos } & 893,5 & 801,1 & 779,7 & 774,7 & 760,0 & 753,4 & 662,3 & 654,1 & 500,1 & 445,4 & 368,2 & 365,2 \\
\hline & 10 & 2o & 3o & $4 \underline{0}$ & 50 & 60 & 70 & $8^{\circ}$ & 9음 & 100 & 110 & $12^{\circ}$ \\
\hline $\begin{array}{l}\text { № de indicadores } \\
\text { superior a } 80 \%\end{array}$ & 8 & 7 & 6 & 7 & 7 & 7 & 6 & 6 & 3 & 3 & 3 & 3 \\
\hline \multirow[t]{2}{*}{ Padrão-ouro } & 1000,0 & 858,9 & 828,8 & 834,7 & 808,6 & 810,2 & 723,3 & 667,7 & 520,2 & 518,6 & 410,6 & 377,9 \\
\hline & 10 & 20 & 40 & 3은 & 60 & 50 & 70 & 80 & 90 & 100 & 110 & $12^{\circ}$ \\
\hline \multirow[t]{2}{*}{ Hieraquização } & 954,1 & 797,7 & 765,0 & 782,6 & 749,0 & 733,2 & 641,6 & 629,8 & 436,9 & 347,7 & 276,5 & 322,3 \\
\hline & 1o & 2o & 40 & 3o & 50 & 60 & 7음 & 8ㅇ & 9o & 100 & $12^{\circ}$ & 119 \\
\hline Classificação & Excelente & \multicolumn{5}{|c|}{ Qualidade boa } & \multicolumn{2}{|c|}{ Q ualidade regular } & \multicolumn{4}{|c|}{ Insatisfatória } \\
\hline $\begin{array}{l}\text { Pontos de corte } \\
\text { propostos }\end{array}$ & acima de $85 \%$ & \multicolumn{5}{|c|}{ entre $70 \%$ e $85 \%$} & \multicolumn{2}{|c|}{ entre $50 \%$ e $70 \%$} & \multicolumn{4}{|c|}{ abaixo de $50 \%$} \\
\hline
\end{tabular}

dos procedimentos necessários para garantir a sobrevida do concepto. O alojamento conjunto e o incentivo ao aleitamento exclusivo, além de seu impacto emocional, com o estabelecimento precoce do vínculo mãe-filho, propõem-sea ser fatores de proteção para o recém-nato em seu primeiro ano de vida. E a consulta de puerpério é a avaliação do desfecho, se o processo se encerrou conforme o esperado.

Estes critérios foram propostos numa tentativa de sumarizar o que seria a avaliação do processo de assistência ao nascimento e parto. Ainda que não o avaliem exaustivamente, acredita-se que, provavelmente, hospitais que apresentaram desempenho inadequado nestes o terão com quaisquer outros grupos de indicadores. Alguns constam de documento da OPAS (Mora et al., 1993), outros foram propostos por outros autores (Bobadilla, 1989) e outros são proposições originais. Entretanto, esta é uma primeira tentativa de organizar um conjunto de indicadores vinculados aos objetivos da assistência, e buscaram-se aqueles que representavam tecnologias de baixo custo e amplo impacto, adequando a seleção ao momento histórico da organização dos serviços de saúde. Temse consciência que, na medida em que certos problemas forem sendo resolvidos, como, por exemplo, a generalização do alojamento conjunto, será necessário substituí-los por outros indicadores que também dêem conta dessa visão de integralidade e sejam discriminantes. Todavia, nesta investigação, estes mostraram consistência em todos os métodos de valida- ção, seja na grosseira seleção por número de variáveis em que apresentavam proporções superiores a $80 \%$, seja quando foi adotado um padrão-ouro, seja quando os indicadores foram hierarquizados. Saliente-se que cinco dos hospitais cumpriram $80 \%$ do realizado pelo padrão-ouro, e que seu processo de assistência foi classificado como bom por praticamente todos os métodos.

Como já foi dito, a avaliação de processo de assistência é procedimento complexo e pode muitas vezes ser contaminada pela subjetividade. Para evitar esta última, ou reduzi-la ao mínimo, é necessário estabelecer padrões de qualidade (Chassin, 1988), como parâmetros para estimar a adequação dos procedimentos. Segundo Donabedian (1982), critérios ou padrões variam em validade, autoridade e vigor, podem ser implícitos ou explícitos e devem derivar de fundamentos cientificamente validados pelo conhecimento. Critérios implícitos ou tácitos são adotados quando, por exemplo, se expõe um caso a um especialista, que utiliza seu conhecimento e experiência pessoais para julgar o processo de assistência. Já critérios ou padrões explícitos para cada categoria de casos são especificados com anterioridade, geralmente com bastante detalhe e por uma junta de especialistas, antes do início da avaliação individual de casos. São, portanto, mais recomendáveis (Chassin, 1988). Visando a reduzir a margem de subjetividade, Lembcke (1967) propôs alguns pontos cardeais para a formulação de critérios explícitos que foram aprofundados 
por Donabedian (1982), quais sejam: a) objetividade, isto é, definição por escrito, com precisão e detalhe, para torná-los relativamente imunes a diferenças entre avaliadores; b) suscetibilidade de verificação, seja por exames de laboratório, consultas, ou documentação; c) uniformidade, ou seja, devem ser aplicáveis independentemente de fatores como tamanho e localização do hospital, qualificações dos médicos e status sócio-econômico do paciente; $d$ ) especificidade, em termos de aplicação específica a uma doença ou procedimento; e) pertinência em relação aos objetivos finais da assistência médica a ser avaliada; f) aceitabilidade, ou seja, deverão estar de acordo com padrões geralmente aceitos de boa qualidade, como os estipulados em livros ou artigos científicos. A presente proposta para avaliação da qualidade de processo acompanhou as seguintes etapas: 1) relacionou os objetivos do programa de assistência materno-infantil; 2) estabeleceu, a partir de normas existentes, consideradas como "fundamentos cientificamente validados pelo conhecimento" (Donabedian, 1982), uma lista de dez potenciais indicadores para qualidade do processo, dentro de uma visão de integralidade da assistência e com uma preocupação de identificar atividades que expressassem atenção tanto ao risco individual como ao coletivo; 3) com esse processo identificou indicadores orientados pelos pontos cardeais de Lembcke, ou seja, indicadores: a) objetivos, já que por serem explicitados afastam o "achismo", a subjetividade; b) suscetíveis de verificação, pois baseiam-se em dados passíveis de ser verificados por auditoria de prontuários; c) uniformes, pois podem ser aplicados a todos os hospitais, independentemente de seu porte, demanda ou complexidade; d) específicos para a assistência ao nascimento e parto; e) pertinentes aos objetivos do Programa de Assistência Materno-Infantil; f) aceitáveis, já que se baseiam em normas técnicas, aquilo que é preconizado ou recomendado.

Há algumas considerações sobre a prática proposta. A literatura sobre avaliação de serviços tem crescido, e há, hoje em dia, um movimento social em busca da qualidade, em todos os ramos da atividade humana. Nesse contexto, não há como excluir a atividade médica, eé importante que os profissionais de saúde não apenas se acostumem com as avaliações de seu desempenho, como também, sempre que possível, participem da elaboração dos critérios de avaliação e de todo o processo de implantação de programas de garantia de qualidade. Outra das necessidades que a modernização dos serviços impõe é a de atualização do sistema de informações, e o Sistema Informático Perinatal utilizado apresenta algumas vantagens nesse sentido, como: a) a adoção de um formulário previamente sistematizado, como a HCPS, pode servir de lembrete para os profissionais que vão preenchê-lo, para que não omitam ou esqueçam informações; b) a elaboração do formulário pelo Centro Latino-Americano de Perinatologia - CLAP/OPAS/OMS - contou com a colaboração de inúmeros especialistas de obstetrícia, neonatologia, perinatologia, sistemas de informação etc., traduzindo, portanto, esse conhecimento acumulado; c) o programa para entrada e manuseio dos dados em computador já está elaborado e é constantemente aprimorado, sendo de distribuição gratuita; d) como foi constatado por este trabalho, a mensuração do grau de completude de alguns dados, e, portanto, uma avaliação da qualidade do processo torna-se possível.

Cabem alguns comentários sobre as limitações do presente estudo. Em vista de os hospitais terem integrado o projeto de pesquisa através de convite, não se trata de uma amostra probabilística, não sendo os resultados, portanto, generalizáveis. Entretanto, são 12 estudos de caso que permitiram uma série de inferências quanto à qualidade da assistência prestada. Também são importantes algumas considerações sobre o registro de dados. Nos melhores hospitais, os procedimentos são realizados e registrados. Pode-se supor que a ausência de registro de, por exemplo, sorologia para lues, tipagem sanguínea, boletim de Apgar, avaliação de idade gestacional por exame físico, significa a não-realização dos procedimentos, se a coleta de dados foi correta e a supervisão dessa coleta foi rigorosa, como o foi neste trabalho. Assim, considerou-se que dados inscritos nos prontuários representam a realização e registro dos procedimentos, e, embora aceite-se a crítica de Vuori (1991) de "desenvolver critérios e padrões somente para os aspectos do atendimento que são sabidamente registrados", observou-se que nem mesmo esses dados estão sempre presentes.

Este trabalho, longe de apenas responder às necessidades de avaliações, permitiu constatar que há muitas perspectivas de estudos que se abrem para o futuro. Em nível da teoria, é necessário aprofundar a reflexão sobre o referencial teórico e as várias unidades de análise possíveis, incorporando a abordagem epistemológica proposta por Samaja (1994). Também é importante tecer considerações sobre o significado dos resultados, e identificar quais os que melhor traduziriam o impacto de um atendimento com qualidade. Uma outra possibilida- 
de é a realização de pesquisa visando a relacionar estrutura, processo e resultado, mas queleve em conta o contexto.

Em nível da prática, também há várias perspectivas. Por exemplo, na medida em que normas representam o saber científico reconhecido no atual avanço da ciência e tecnologia, e este conhecimento é dinâmico, é necessário que as normas técnicas sejam periodicamente atualizadas. Em conseqüência, indicadores que são derivados de normas também deverão ser aprimorados periodicamente. Outra derivação possível é a incorporação dessa preocupação com qualidade da assistência na rotina dos serviços,

\section{Agradecimentos}

A Maria Lúcia Lebrão, orientadora da dissertação que deu origem a este trabalho. $E$ às boas colaborações de Isabel Cristina M. de Freitas, Marcello Di Pietro, Conceição Segre, Solum Donas, Bruce Duncan, César Victora, Marina F. Réa, Nelson Rodrigues dos Santos, Sonia Nussenzweig Hotimsky, Sandra Musa e das bibliotecárias da Faculdade de Saúde Pública-USP, recebidas nos vários momentos de sua elaboração. ou seja, a implantação de programas de garantia de qualidade autogeridos pelos serviços. Esta certamente demandará uma modernização no sistema de informações, de modo que outra possibilidade de desenvolvimento é a adoção mais generalizada do Sistema Informático Perinatal, elaborado pelo Centro Latino-Americano de Perinatologia. Finalmente, há a possibilidade de este trabalho sugerir que muitos se aventurem no desenvolvimento de indicadores de qualidade de processo para outros tipos de assistência, pois, parafraseando Lowry (1988), a proposta deste trabalho é: "Não combata os indicadores, use-os" e, acrescente-se, "melhore-os".

\section{Referências}

BELIZAN, J. M.; DIAZ, A. G.; GIACOMINI, H.; HORCHER, R.; MARTELL, M.; ONETO, M.; QUARANTIA, P. \& SCHWARCZ, R. L., 1976. Historia Clínica Perinatal - Propuesta de un Modelo. Buenos Aires: Ministerio de Bien Estar Social/OPS/OMS.

BOBADILLA, J., 1989. La salud perinatal y la calidad de la atención médica en la ciudad de Mexico. In: Salud, Enfermedad y Muerte delos Niños en Amé rica Latina (A. Lattes, M. Farren \& J. MacDonald, orgs.), pp. 161-180, Ottawa: Clacso-IDRC.

CARVALHEIRO, J. R., 1984. Perspectivas de la investigación epidemiológica aplicada a la evaluacion de la salud. In: Seminario sobre Usos y Perspectivas de la Epidemi ología. Buenos Aires, 7 - 10 Noviembre 1983. Publ. OPAS no PNSP - 84-47. Washington: OPAS.

CHASSIN, M. R, 1988. Standards of care in medicine. Inquiry, 25:437-453.

COOKE, R. W. I., 1988. Outcome and costs of care for the very immature infant. British Medical Bulletin, 44:1133-1151.

DIAZ, A. G.; SCHWARCZ, R.; DIAZ ROSSELLO, J. L.; SIMINI, F.; GIACOMINI, H.; LOPEZ, R.; MARTELL, M.; FESCINA, R.; DE MUCIO, B. \& MARTINEZ, G., 1990. Sistema informativo perinatal. Publicación Científica CLAP no 1.203. Montevideo, CLAP/ OPS/OMS. 
DONABEDIAN, A., 1982. Explorations in Quality Assessment and Monitoring- Vol I: The definitions of Quality and Approaches to its Assessment; Vol. II: The Criteria and Standards of Quality. Ann Arbor: Health Administration Press.

DONABEDIAN, A., 1988. The quality of care - how can it be assessed? Journal of the American Medical Association, 260:1.743-1.748.

GARLAND, S. M. \& KELLY, N. V., 1989. Is antenatal screening for syphilis worthwhile? Medical Journal of Australia, 151:368-372.

GENTILE DE MELLO, C., 1977. A epidemiologia da cesárea. In: Saúde e Assistência Médica no Brasil (C. Gentile de Mello, org.), pp. 121-129, São Paulo: CEBES-Hucitec.

GUEDES, J. S., 1972. Contribuição para o Estudo da Evolução do Nível de Saúde do Estado deSão PauIo: Análise das Regiões Administrativas: 19501970. Tese de Doutorado, São Paulo: Faculdade de Saúde Pública, Universidade de São Paulo.

HOLTHOF, B. \& PRINS, P., 1993. Comparing hospital perinatal mortality rates. A quality improvement instrument. Medical Care, 31:801-807.

HULL, V.; THAPA, S. \& WIKNJOSASTRO, G., 1989. Breast feeding and health professionals: a study in hospitals in Indonesia. Social Scienceand Medicine 28:353-364.

LEAPE, L. L., 1989. Unnecessary surgery. Health Services Research, 24:351-407.

LEMBCKE, P. A., 1967. Evolution of the medical audit. Journal of the American Medical Association, 199: 111-118.

LEWIS, C. E., 1974. The state of art of quality assesment -1973. Medical Care, 12:799-806.

LOWRY, S., 1988. Focus on performance indicators. British Medical Journal, 296:992-994.

MORA, G.; BETTS, C.; GAY, J.; HARDEE, K.; CHAMBERS, V. \& FOX, L., 1993. Quality of Care in Women's Reproductive Health: A Framework for Latin America and the Caribbean (Draft). Washingotn, D.C.: PanAmerican Health Organization. (mimeo.)

MPAS (Ministério da Previdência e Assistência Social), 1983. Resolução Inamps no 18/83 de 05/09/83. Programa de Reorientação da Assistência Obstétrica e Pediátrica - Alojamento Conjunto. Brasília: BS/ DG/Inamps (publicação 174 de 13.09.83) e BS/ DG/Inamps (republicação 211 de 08.11 .83 em virtude de incorreções no original).

MS (Ministério da Saúde), 1984a. Textos Bási cos de Saúde - Assistência Integral à Saúde da Mulher: Bases da Ação Programática. Brasília: Centro de Documentação do Ministério da Saúde.

MS (Ministério da Saúde), 1984b. Textos Bási cos de Saúde - Assistência Integral à Saúde da Criança: Ações Básicas. Brasília: Centro de Documentação do Ministério da Saúde.

MS (Ministério da Saúde), 1986. Portaria 542 de 22/12/86. Brasília.

MS (Ministério da Saúde), 1991a. Programa de Assistência Integral à Saúde da Mulher (PAISM) Gestação de Alto Risco. Brasília: Normas e Manuais Técnicos (brochura).
MS (Ministério da Saúde), 1991b. Programa de Assistência Integral à Saúde da Mulher (PAISM) Assistência Institucional ao Parto, ao Puerpério e ao Recém-Nascido. Brasília: Normas e Manuais Técnicos (brochura).

MS (Ministério da Saúde), 1993. Portaria no 31, de 15/02/1993. Brasília: Diário Oficial da União, $17 / 02 / 1993$

NIELSEN, T. \& HOKEGARD, K. H., 1984. Cesarean section and intraoperative surgical complications. Acta Obstetricia et Gynecologica Scandinavica, 63: 103-108.

OMS/ OPAS (Organização Mundial de Saúde/ Organização Pan-Americana da Saúde), 1987. Condições deEficiência nos Serviços de Atenção Materno-Infantil. Fascículo II. Brasília: Organização PanAmericana da Saúde. (mimeo.)

PLAUT, R., 1984. Analisis de riesgo. Alcance y limitaciones para el administrador de salud. Boletin de la Oficina Sanitaria Panamericana, 96:296-304.

RATTNER, D. \& SEGRE, C. A. M., 1990. Relatório da pesquisa "História Clínica Perinatal Simplificada: É viável em São Paulo ?" São Paulo: Instituto de Saúde. (mimeo.)

RATTNER, D., 1991. Subsídios para a Avaliação da Qualidade do Processo de Assistência ao Parto. Dissertação de Mestrado, São Paulo: Faculdade de Saúde Pública, Universidade de São Paulo.

RATTNER, D., 1996. Sobre a hipótese de estabilização das taxas de cesárea no Estado de São Paulo, Brasil. Revista de Saúde Pública, 30:19-33.

REIS, O. M., 1971. Planejamento Sócio-Econômico. Aplicação da Metodologia a um Setor Social. O Caso do Setor Saúde. Brasília: Ministério do Planejamento e Coordenação Geral/Instituto de Planejamento Econômico e Social (Ipea)/Centro de Treinamento e Pesquisa para o Desenvolvimento Econômico (Cendec). Documento de trabalho para os cursos Cendec. (mimeo.)

SAM AJA, J., 1994. Epistemología y Metodología. Elementos para una Teoría de la Investigación Científica. Buenos Aires: Eudeba-Editora Universitaria de Buenos Aires.

SES/ SP (Secretaria de Estado da Saúde de São Paulo), (s/ data). Norma Técnica para Instalação e Funci onamento de Berçário em Hospital. São Paulo: Instituto de Saúde. (mimeo.)

SES/SP (Secretaria de Estado da Saúde de São Paulo), 1975. Programa de Assistência à Criança. São Paulo: Secretaria de Estado de Saúde (mimeo.)

SES/ SP (Secretaria de Estado da Saúde de São Paulo), 1984. Normas Básicas para Alojamento Conjunto. São Paulo: Divisão de Normas Técnicas. Publicado no Diário Oficial em 07.09.84, pp.15-16.

SES/SP (Secretaria de Estado da Saúde de São Paulo), 1985. Subprograma da Saúde da Mulher Vol. II: Pré-Natal Normal; Vol III: Pré-Natal Patológico. São Paulo: Grupo de Saúde da Mulher.

SES/ SP (Secretaria de Estado da Saúde de São Paulo), 1989. Resolução 143 de 18/ 08/ 89. São Paulo: Diário Oficial, 19.08.89. 
SES/ SP (Secretaria de Estado da Saúde de São Paulo), 1994. Norma do Programa de Imunização. São Paulo: Comissão Permanente de Assessoria em Imunizações. (mimeo.)

SIMINI, F.; SCHWARCZ, R.; DIAZ, A. C.; LOPEZ, R.; BELITZKY, R.; TENZER, S. M. \& FESCINA, R., 1989. Sistema Informático Perinatal - Manual de Uso de los Programas. Centro Latinoamericano de Perinatología y Desarrollo Humano - CLAP/ OPS/OMS.

THOMPSON, J. D.; MARQUIS, D. B.; WOODWARD, R. L. \&YEOMANS, R. C., 1968. End-result measurements of the quality of obstetrical care in two U.S. Air Force Hospitals. Medical Care, 6:131-143.

TOKUHATA, G. K.; COLFLESH, V. G.; RAM ASWAMY, K.; MANN, L. A. \& DIGON, E., 1973. Hospital and related characteristics associated with perinatal mortality. American Journal of Public Health, 63: 227-237.

VUORI, H., 1980. Optimal and logical quality: two neglected aspects of the quality of health services. Medical Care, 28:975-985.

VUORI, H., 1991. A qualidade da saúde. Divulgação em Saúde para Debate, 3: Caderno de Ciência e Tecnologia 1:17-25.
WILLIAMS, R. L., 1979. Measuring the effectiveness of perinatal medical care. Medical Care, 12:95110.

WILLIAMS, W. W.; HICKSON, M. A.; KANE, M. A.; KENDAL, A. P.; SPIKA, J. S. \& HINMAN, A. R., 1988. Immunization policies and vaccine coverage among adults - the risk for missed opportunities. Annals of Internal Medicine, 108:616-625.

WHO (World Health Organization), 1982. Quality Assurance of Health Services. Geneve: Series Public Health in Europe no 16. Regional Office for Europe.

WHO (World Health Organization), 1986. Infant and Young Child Nutrition (Progress and Evaluation Report and Status of Implementation of the International Code of Marketing of Breast Milk Substitutes). Guidelines Concerning the Main Health and Socio-Economic Circumstances in Which Infants Have to Be Fed of Breast Milk Substitutes. Geneve: A 39/ 8 Add. 1. World Health Organization - Thirty Ninth World Assembly. (mimeo.) 